\title{
Cancer relevance of signal recognition particle RNA and other non-coding RNAs in extracellular vesicles
}

\author{
Germana Rappa ${ }^{1}$, Alice Conigliaro ${ }^{2}$, Mark F. Santos ${ }^{1}$, Riccardo Alessandro ${ }^{2}$, Aurelio Lorico ${ }^{1}$ \\ ${ }^{1}$ Department of Pathology and Cancer Research Program, Roseman University College of Medicine, Las Vegas, USA; ${ }^{2}$ Department of Biopathology \\ and Medical Biotechnology, University of Palermo, Palermo, Italy \\ Correspondence to: Aurelio Lorico, MD, PhD. Associate Professor of Pathology and Co-Director, Cancer Research Program, Roseman University College \\ of Medicine, 10530 Discovery Drive, Las Vegas, NV 89135, USA. Email: alorico@roseman.edu; Riccardo Alessandro, PhD. Director, Department of \\ Biopathology and Medical Biotechnology, University of Palermo, Via Divisi 83, Palermo 90100, Italy. Email: riccardo.alessandro@unipa.it. \\ Comment on: Nabet BY, Qiu Y, Shabason JE, et al. Exosome RNA Unshielding Couples Stromal Activation to Pattern Recognition Receptor \\ Signaling in Cancer. Cell 2017;170:352-366.e13.
}

Submitted Sep 15, 2017. Accepted for publication Sep 19, 2017.

doi: $10.21037 /$ tcr.2017.09.38

View this article at: http://dx.doi.org/10.21037/tcr.2017.09.38

Exosomes and ectosomes are membrane-bound nanobiological units released from most cell types into the extracellular milieu. Currently, they are distinguished mainly by their biogenesis, exosomes being released upon fusion of multi-vesicular bodies with the plasma membrane and ectosomes budding directly from it (1). Given the experimental difficulty to identify and/ or isolate pure populations of exosomes and ectosomes, here we will refer to them collectively as extracellular vesicles (EVs). The physiological functions of EVs are diverse (e.g., cell-cell communication, cellular differentiation, immunity, inflammation) (2-6). In cancer, aberrant activity of the export machinery results in the release of a number of key proteins and nucleic acids associated with EVs. These EVs can modify the normal cellular microenvironment by promoting the formation of the pre-metastatic niche, modulate and/ or interfere with the immunologic properties of bone marrow-derived multipotent mesenchymal stromal cells (MSCs), a major constituent of cellular microenvironment invaded by cancer cells, and interact with B cells to foster tumor-promoting humoral immunity, ultimately playing a fundamental role in cancer progression $(2,7,8)$.

In the Cell paper "Exosome RNA unshielding couples stromal activation to pattern recognition receptor signaling in cancer", Nabet and colleague (9) bring to the limelight the relatively new concept of RNA unshielding, in particular for signal recognition particle (SRP) RNA, coupled with EV-mediated cell transfer. The importance of SRPs, highly conserved complexes of a 7S RNA and six proteins that bind to pattern recognition receptors (PRRs), has been recently recognized because of in-depth mechanistic investigation and for their potential role in cancer progression (10). Specifically, Nabet and colleagues (9) reported that unshielded RN7SL1 noncoding (nc) SRP RNA transferred by activated stromal EVs to adjacent triple-negative breast cancer cells stimulated the viral RNA PRR RIG-I, resulting in enhanced tumor growth and a malignant phenotype. We propose for this EV-mediated mechanism the acronym EVAMR for $\mathrm{EV}$ associated molecular response. Their data reinforce previous studies on the role of EVs in the crosstalk between stromal cells and breast cancer cells (11). Cancer cells are not alone during the process of invasion. They live in a crowded environment where an intense bi-directional communication with "normal" cells is incessantly occurring. Cancer-associated fibroblasts, endothelial cells, macrophages, immune-derived cells, and MSCs have been described to interact with malignant cells and are potentially able to affect the malignant phenotype. Similarly, melanoma-derived EVs from the primary tumor reportedly "educate" their environment to form a pro-tumorigenic niche and program bone marrow-derived progenitors at the pre-metastatic site to assume a proangiogenic phenotype, thereby enhancing metastatic dissemination (2). 
The prevalence of ncRNA species distinct from miRNAs, in particular SRP RNA, found by Nabet and colleagues (9) in stromal cell-derived EVs released during co-culture with triple negative breast cancer cells, is surprising given the general consensus in the EV field on the prevalence of EVassociated coding RNA and miRNA. EVs are composed of lipids, proteins, nucleic acids and other molecules. Among nucleic acids, miRNAs have been studied more in depth, with several groups reporting that miRNAs isolated from EVs are enriched relative to the miRNAome of the originating cells (12-16), indicating that RNA molecules are selectively incorporated into EVs. From the pioneering study of Valadi and colleagues (12), a number of studies have been published to date demonstrating that miRNA secretion by EVs is not only a mechanism whereby cells rapidly dispose of miRNAs in excess to maintain RNA homeostasis, but also a strategy for the horizontal transfer of RNAs (17). Today we know that miRNAs, independent from the cellular amount, are selectively retained within the cells or exported $(18,19)$, often together with the molecular machinery required to induce a miRNA-mediated silencing so to "guarantee" their activity once they reached their destination (20). EV-associated miRNAs have clinical potential both as therapeutic targets and biomarkers for diagnosis and monitoring of several diseases (21). To date in the main EV data repository "Vesiclopedia" (www. microvesicles.org), there are $~ 28,000$ entries for mRNAs and 5,000 entries for miRNAs, but no entries for other ncRNAs. Newcomers to the EV field searching the web for "ncRNAs" and "exosomes" will likely encounter as one of the first "hits" a misleading review titled "Noncoding RNAs in EVs: New Players in Cancer Biology" (Curr Genomics 2015), where the authors devote the whole article to miRNAs, failing to discuss studies on the presence of other types of ncRNA in EVs. Intriguingly, most studies on the characterization of EV-associated RNA have employed microarray or real time quantitative PCR (qPCR) assays; but because of the inherent limitations of these technologies, mostly miRNAs and mRNAs have been detected. However, new exciting data demonstrate the ability of EVs to carry a multitude of other ncRNA families whose identification may provide a more accurate snapshot of the status of the parental cell.

The growing use of tools like next-generation sequencing and deeper analysis of EV content, in terms of nucleic acids, reveal that the majority of normal and cancer cells load different RNA species with a size distribution between 25 and 700 nucleotides (nt) in EVs. Small size RNAs (<700 nt) were found in saliva and breast milk EVs (22). EVs released by human MSCs (23) were found to contain smaller RNA species (<500 nt in length), while the majority of RNA in immune cell derived EVs were reportedly shorter than $200 \mathrm{nt}$ (14). Even if different EV isolation methods may account for the size discrepancies among the small ncRNA families (24), miRNA, tRNA, rRNA, small nuclear RNA (snRNA), small nucleolar RNA (snoRNA) and piwiinteracting RNA (piRNA) have been found in most studies $(14,25)$. Intriguingly, the contradictory data concerning the relative percentage of small ncRNA in EVs, confirm the large heterogeneity in EV preparations (I) among the different parental cells; (II) in the same producing cell $(22,26)$. Moreover, as shown by Nabet et al. (9), the various small ncRNA content in EVs is strongly conditioned by the micro-environment.

The EV world is still in its infancy. The spatiotemporal fate of EVs in vivo is currently obscure (27), and their intracellular path as well as the targets of the EV-associated biomaterials are still elusive. Moreover, the distinction of sub-classes of exosomes and ectosomes is still unclear. A very high degree of $\mathrm{EV}$ heterogeneity is indicated by the observation that, although the cargo space of an individual vesicle is very limited, thousands of proteins and RNA species are usually identified in an EV preparation from a monoculture. A single cell can release EVs of varying biogenesis, size, and cargo and different cell types may produce distinct, cell or tissue-specific repertoires of EVs.

The recent discovery by one of our labs of spathasomes, which are sub-nuclear compartments originating from subdomains of Rab7 $7^{+}$late endosomes and nuclear envelope invaginations and are able to deliver $\mathrm{EV}$-derived biomaterials into the nuclear compartment (28), opens the possibility that $\mathrm{EV}$ ncRNAs are at least in part destined to the nucleus of the recipient cell. Interestingly, ncRNAs regulate gene expression at the nuclear level. In particular, SRP RNAs are produced at the nucleolar or peri-nucleolar level and complete SRPs are assembled at the same location (29).

Bringing SRP RNA and other non-miRNA species of ncRNA into the field of EVs as new important molecular players invites many new important questions: (I) are the SRP RNAs depending on the different releasing stromal cells? For example, can the pathological invasion of endothelial cells during tumoral angiogenesis, resembling the physiological invasion occurring during inflammation, be affected by these ncRNAs? Although several evidences have indicated that EVs released from cancer cells or from stromal cells are involved in the modulation of 
neoangiogenesis (30), no data are available on the quality of EVs released from co-culture of endothelial cells with cancer cells and on the ratio between the different types of ncRNA. The picture is further complicated by the evidence that oncogene activation alters secretion of ncRNA via EVs by modifying the interaction of AGO2 with endosomes (31). Moreover, environmental components of cancer development such as hypoxia or metabolic shifts may add another, still unknown, level of intricacy; (II) are the results from Nabet's paper exploitable for other cancer histotypes and is this mechanism involved in metastatic niche formation and maintenance? Up to know only few papers have focused on this aspect of EV biology and triplenegative breast cancer cells have been used as model. It will be interesting to investigate if similar mechanisms do exist in other deadly cancers as pancreatic cancer, colon cancer or in hematopoietic malignancies, where the critical role played by MSCs in the maintenance of malignancy and in drug-resistance phenotype is well established (32); (III) in addition to the direct contact activation of stromal cells, what is the role, if any, of cancer EVs on stromal cells? (IV) Is the high level of SRP RNA only present in co-culture EVs or also in single stromal or cancer cell cultures? (V) What is the relevance of stromal delivery of non-miRNA ncRNA to cancer cells of patients with aggressive tumors?

Through the paper by Nabet and colleague (9) and the growing interest for $\mathrm{EV}$-associated ncRNA in cancer, a very exciting field of investigation unwraps.

\section{Acknowledgments}

Funding: None.

\section{Footnote}

Provenance and Peer Review: This article was commissioned and reviewed by the Section Editor Sheng Sun (Massachusetts General Hospital Cancer Center, Harvard Medical School, Boston, MA, USA).

Conflicts of Interest: All authors have completed the ICMJE uniform disclosure form (available at http://dx.doi. org/10.21037/tcr.2017.09.38). The authors have no conflicts of interest to declare.

Ethical Statement: The authors are accountable for all aspects of the work in ensuring that questions related to the accuracy or integrity of any part of the work are appropriately investigated and resolved.

Open Access Statement: This is an Open Access article distributed in accordance with the Creative Commons Attribution-NonCommercial-NoDerivs 4.0 International License (CC BY-NC-ND 4.0), which permits the noncommercial replication and distribution of the article with the strict proviso that no changes or edits are made and the original work is properly cited (including links to both the formal publication through the relevant DOI and the license). See: https://creativecommons.org/licenses/by-nc-nd/4.0/.

\section{References}

1. György B, Szabó TG, Pásztói M, et al. Membrane vesicles, current state-of-the-art: emerging role of extracellular vesicles. Cell Mol Life Sci 2011;68:2667-88.

2. Peinado H, Alečković $M$, Lavotshkin $S$, et al. Melanoma exosomes educate bone marrow progenitor cells toward a pro-metastatic phenotype through MET. Nat Med 2012;18:883-91.

3. Pucci F, Garris C, Lai CP, et al. SCS macrophages suppress melanoma by restricting tumor-derived vesicle-B cell interactions. Science 2016;352:242-6.

4. Bakhti M, Winter C, Simons M. Inhibition of myelin membrane sheath formation by oligodendrocyte-derived exosome-like vesicles. J Biol Chem 2011;286:787-96.

5. Ratajczak J, Wysoczynski M, Hayek F, et al. Membranederived microvesicles: important and underappreciated mediators of cell-to-cell communication. Leukemia 2006;20:1487-95.

6. Janowska-Wieczorek A, Majka M, Kijowski J, et al. Platelet-derived microparticles bind to hematopoietic stem/progenitor cells and enhance their engraftment. Blood 2001;98:3143-9.

7. Zhang HG, Grizzle WE. Exosomes: a novel pathway of local and distant intercellular communication that facilitates the growth and metastasis of neoplastic lesions. Am J Pathol 2014;184:28-41.

8. Correa D, Somoza RA, Lin P, et al. Mesenchymal stem cells regulate melanoma cancer cells extravasation to bone and liver at their perivascular niche. Int J Cancer 2016;138:417-27.

9. Nabet BY, Qiu Y, Shabason JE, et al. Exosome RNA Unshielding Couples Stromal Activation to Pattern Recognition Receptor Signaling in Cancer. Cell 2017;170:352-366.e13.

10. Akopian D, Shen K, Zhang X, et al. Signal recognition 
particle: an essential protein-targeting machine. Annu Rev Biochem 2013;82:693-721.

11. Luga V, Zhang L, Viloria-Petit AM, et al. Exosomes mediate stromal mobilization of autocrine WntPCP signaling in breast cancer cell migration. Cell 2012;151:1542-56.

12. Valadi H, Ekström K, Bossios A, et al. Exosome-mediated transfer of mRNAs and microRNAs is a novel mechanism of genetic exchange between cells. Nat Cell Biol 2007;9:654-9.

13. Skog J, Würdinger T, van Rijn S, et al. Glioblastoma microvesicles transport RNA and proteins that promote tumour growth and provide diagnostic biomarkers. Nat Cell Biol 2008;10:1470-6.

14. Nolte-'t Hoen EN, Buermans HP, Waasdorp M, et al. Deep sequencing of RNA from immune cell-derived vesicles uncovers the selective incorporation of small noncoding RNA biotypes with potential regulatory functions. Nucleic Acids Res 2012;40:9272-85.

15. Rappa G, Mercapide J, Anzanello F, et al. Biochemical and biological characterization of exosomes containing prominin-1/CD133. Mol Cancer 2013;12:62.

16. Raposo G, Stoorvogel W. Extracellular vesicles: exosomes, microvesicles, and friends. J Cell Biol 2013;200:373-83.

17. Squadrito ML, Baer C, Burdet F, et al. Endogenous RNAs modulate microRNA sorting to exosomes and transfer to acceptor cells. Cell Rep 2014;8:1432-46.

18. Guduric-Fuchs J, O'Connor A, Camp B, et al. Selective extracellular vesicle-mediated export of an overlapping set of microRNAs from multiple cell types. BMC Genomics 2012;13:357.

19. Pigati L, Yaddanapudi SC, Iyengar R, et al. Selective release of microRNA species from normal and malignant mammary epithelial cells. PLoS One 2010;5:e13515.

20. Melo SA, Sugimoto H, O'Connell JT, et al. Cancer exosomes perform cell-independent microRNA biogenesis and promote tumorigenesis. Cancer Cell 2014;26:707-21.

21. Thind A, Wilson C. Exosomal miRNAs as cancer biomarkers and therapeutic targets. J Extracell Vesicles

Cite this article as: Rappa $\mathrm{G}$, Conigliaro A, Santos MF, Alessandro R, Lorico A. Cancer relevance of signal recognition particle RNA and other non-coding RNAs in extracellular vesicles. Transl Cancer Res 2017;6(Suppl 7):S1257-S1260. doi: 10.21037/tcr.2017.09.38
2016;5:31292.

22. Lässer C, Alikhani VS, Ekström K, et al. Human saliva, plasma and breast milk exosomes contain RNA: uptake by macrophages. J Transl Med 2011;9:9.

23. Chen $W$, Huang $Y$, Han J, et al. Immunomodulatory effects of mesenchymal stromal cells-derived exosome. Immunol Res 2016;64:831-40.

24. Huang X, Yuan T, Tschannen M, et al. Characterization of human plasma-derived exosomal RNAs by deep sequencing. BMC Genomics 2013;14:319.

25. Liu Y, Gu Y, Han Y, et al. Tumor Exosomal RNAs Promote Lung Pre-metastatic Niche Formation by Activating Alveolar Epithelial TLR3 to Recruit Neutrophils. Cancer Cell 2016;30:243-56.

26. Lässer C, Shelke GV, Yeri A, et al. Two distinct extracellular RNA signatures released by a single cell type identified by microarray and next-generation sequencing. RNA Biol 2017;14:58-72.

27. van Balkom BW, Eisele AS, Pegtel DM, et al. Quantitative and qualitative analysis of small RNAs in human endothelial cells and exosomes provides insights into localized RNA processing, degradation and sorting. J Extracell Vesicles 2015;4:26760.

28. Rappa G, Santos MF, Green TM, et al. Nuclear transport of cancer extracellular vesicle-derived biomaterials through nuclear envelope invagination-associated late endosomes. Oncotarget 2017;8:14443-61.

29. Alavian CN, Politz JC, Lewandowski LB, et al. Nuclear export of signal recognition particle RNA in mammalian cells. Biochem Biophys Res Commun 2004;313:351-5.

30. Kholia S, Ranghino A, Garnieri P, et al. Extracellular vesicles as new players in angiogenesis. Vascul Pharmacol 2016;86:64-70.

31. McKenzie AJ, Hoshino D, Hong NH, et al. KRAS-MEK Signaling Controls Ago2 Sorting into Exosomes. Cell Rep 2016;15:978-87.

32. Bergfeld SA, DeClerck YA. Bone marrow-derived mesenchymal stem cells and the tumor microenvironment. Cancer Metastasis Rev 2010;29:249-61. 